\title{
Article
}

\section{Differential Games for an Infinite 2-Systems of Differential Equations}

\author{
Muminjon Tukhtasinov ${ }^{1,+}{ }^{\text {, Gafurjan Ibragimov }}{ }^{2,+} \mathbb{C}^{\mathbb{D}}$, Sarvinoz Kuchkarova ${ }^{1,+}$ and Risman Mat Hasim ${ }^{2, *}$ \\ 1 National University of Uzbekistan, University Street, Al-Mazar District, Tashkent 1000174, Uzbekistan; \\ mumin51@mail.ru (M.T.); kuchkarova11@yandex.ru (S.K.) \\ 2 Department of Mathematics, Faculty of Science, University Putra Malaysia, Serdang 43400, Malaysia; \\ ibragimov@upm.edu.my \\ * Correspondence: risman@upm.edu.my \\ + These authors contributed equally to this work.
}

check for

updates

Citation: Tukhtasinov, M.; Ibragimov,

G.; Kuchkarova, S.; Mat Hasim, R. Differential Games for an Infinite 2-Systems of Differential Equations. Mathematics 2021, 9, 1467. https:// doi.org/10.3390/math9131467

Academic Editor: Vladimir Mazalov

Received: 17 May 2021

Accepted: 6 June 2021

Published: 23 June 2021

Publisher's Note: MDPI stays neutral with regard to jurisdictional claims in published maps and institutional affiliations.

Copyright: (C) 2021 by the authors. Licensee MDPI, Basel, Switzerland. This article is an open access article distributed under the terms and conditions of the Creative Commons Attribution (CC BY) license (https:// creativecommons.org/licenses/by/ $4.0 /)$.

\begin{abstract}
A pursuit differential game described by an infinite system of 2-systems is studied in Hilbert space $l_{2}$. Geometric constraints are imposed on control parameters of pursuer and evader. The purpose of pursuer is to bring the state of the system to the origin of the Hilbert space $l_{2}$ and the evader tries to prevent this. Differential game is completed if the state of the system reaches the origin of $l_{2}$. The problem is to find a guaranteed pursuit and evasion times. We give an equation for the guaranteed pursuit time and propose an explicit strategy for the pursuer. Additionally, a guaranteed evasion time is found.
\end{abstract}

Keywords: pursuer; evader; constraints; strategy

\section{Introduction}

The notion of differential games was introduced by Isaacs [1]. Differential games were developed by Bercovitz [2], Elliot and Kalton [3], Fleming [4,5], Friedman [6], Hajek [7], Ho, Bryson, and Baron [8], Pontryagin [9], Krasovskii [10], Petrosyan [11], Pshenichnyi [12], Subbotin [13], Chikrii [14], and others.

There are many works which deal with the infinite dimensional spaces, such as [15-19]. Differential games studied in the works [17,19-22] are devoted to constructing of the optimal strategies of players.

In the paper of Satimov and Tukhtasinov [23], pursuit and evasion differential games were studied for the parabolic equation. Various cases of control constraints (integral, geometric) were analyzed. Two sets were specified such that pursuit can be completed if the initial state belongs to the first set, and evasion is possible if the initial state belongs to the second set. Note that, the works [24,25] also relates to differential games described by PDE.

In the game with countably many pursuers studied Ibragimov et al. [17] in the Hilbert space $l_{2}$, the duration of the game is prescribed. A formula for the value of the game was found and optimal strategies of the players were constructed explicitly. In the work by Salimi and Ferrara [26], an optimal approach of a finite or denumerable pursuers to one evader is studied. In that paper a formula for the value of the game and optimal strategies of players are proposed. A time-optimal problem of transition of the state of system into the origin was studied by Azamov and Ruziboev [27]. The main result of that paper is estimate of the optimal time from above.

In the present paper, we study differential game problems described by an infinite system of 2-systems of differential equations. We find a guaranteed pursuit time for the pursuit differential game and a guaranteed evasion time for the evasion differential game.

\section{Motivation}

The work [28] the first paper on time-optimal control problem for the parabolic type partial differential equations. The optimal control problems in systems with distributed 
parameters is widely studied [29]. Interesting results were obtained by Albeverio and Alimov [30] for a time-optimal control problem for the parabolic differential equation where the control function is defined on the boundary, and by Chaves-Silva et al. [31] for the null controllability of evolution equations with memory terms, and by Philippe Martin et al. [32] for the structurally damped wave equation where the null controllability holds in some suitable Sobolev space and after a fixed positive time independent of the initial conditions.

Differential game problems described by partial differential equations are considered for the first time in the works $[33,34]$. One of the main tools in studying control or/and differential game problems for the systems described by partial differential equations is the method of Fourier. We can use this method to reduce differential game problems described by partial differential equations to differential game problems described by an infinite system of differential equations [23-25,27,35-40].

Indeed, let a controlled distributed system be described by the following parabolic equation

$$
\frac{\partial z}{\partial t}+A z=w, \quad z(x, 0)=z_{0}(x), x \in D, \quad z(x, t)=0, x \in \partial D, 0<t<T,
$$

where $z=z(x, t)$ is the state of the system, $z_{0}(x) \in L_{2}(D), x=\left(x_{1}, x_{2}, \ldots, x_{n}\right) \in D \subset \mathbb{R}^{n}$, $n \geq 1, D$ is a bounded domain, and it is assumed that the boundary $\partial D$ of $D$ is piecewise smooth, $t \in[0, T]$, and $T>0$ is a given number, $w=w(x, t), w(x, t) \in L_{2}\left(C_{T}\right)$, is the control function, $C_{T}$ is the following open cylinder

$$
C_{T}=\{(x, t) \mid x \in D, 0<t<T\} \subset \mathbb{R}^{n+1},
$$

operator $A z$ is defined by the equation

$$
A z=-\sum_{i, j=1}^{n} \frac{\partial}{\partial x_{i}}\left(a_{i j}(x) \frac{\partial z}{\partial x_{j}}\right), a_{i j}(x)=a_{j i}(x),
$$

$a_{i j}(x)$ are assumed to be bounded measurable functions. Additionally, $\sum_{i, j=1}^{n} a_{i j}(x) \eta_{i} \eta_{j} \geq$ $k \sum_{i=1}^{n} \eta_{i}^{2}$ for all $\left(\eta_{1}, \eta_{2}, \ldots, \eta_{n}\right) \in \mathbb{R}^{n}, x \in D$, and for some positive number $k$.

Then, for any $w(x, t) \in L_{2}\left(C_{T}\right)$ and $z_{0}(x) \in L_{2}(D)$, problem (1) has the only generalized solution $z=z(x, t)$ in the set $\grave{W}_{2}^{1,0}\left(C_{T}\right)$ [41]. Moreover, the solution can be represented in the form ([41], III.3)

$$
z(x, t)=\sum_{i=1}^{\infty} z_{i}(t) v_{i}(x)
$$

where $z_{i}(t), t \in[0, T], i=1,2, \ldots$, are solutions of the following initial value problems

$$
\dot{z}_{i}=\lambda_{i} z_{i}+w_{i}(t), \quad z_{i}(0)=z_{i 0}, \quad i=1,2, \ldots,
$$

the coefficients $\lambda_{1}, \lambda_{2}, \ldots, \lambda_{i}, \ldots$ are positive and they are the generalized eigenvalues of the operator $A$ [37], and $\lambda_{i} \rightarrow+\infty$ as $i \rightarrow \infty, v_{1}(x), v_{2}(x), \ldots, v_{i}(x), \ldots$ are the generalized eigenfunctions of $A$, which form a complete orthonormal system in $L_{2}(D)$, and $w_{i}(t)$ and $z_{i 0}$ are the Fourier coefficients of of the functions $w(x, t)$ and $z_{0}(x)$, respectively, relative to the system $\left\{v_{i}(x)\right\}$,

$$
w(x, t)=\sum_{i=1}^{\infty} w_{i}(t) v_{i}(x), z_{0}(x)=\sum_{i=1}^{\infty} z_{i 0} v_{i}(x) .
$$

Additionally, the series (2) uniformly converges in $L_{2}\left(C_{T}\right)$, and its sum $z(x, t)$ belongs to the Sobolev space $W_{2}^{1}(D)$ for every $t, 0 \leq t \leq T$, and is a continuous in $t$ in $W_{2}^{1}(D)$ [41]. 
For example, in the works $[23,24,38,39]$, a differential game for a PDE of the form

$$
\frac{\partial z}{\partial t}=A z+u-v, \quad A z=-\sum_{i, j=1}^{n} \frac{\partial}{\partial x_{i}}\left(a_{i j}(x) \frac{\partial z}{\partial x_{i}}\right),
$$

was studied by reducing to the following infinite system

$$
\dot{z}_{k}+\lambda_{k} z_{k}=u_{k}-v_{k}, k=1,2, \ldots,
$$

where $u_{k}$ and $v_{k}$ are control parameters of pursuer and evader, respectively, $z_{k}, u_{k}, v_{k} \in \mathbb{R}$, and coefficients $\lambda_{k}, k=1,2, \ldots$, satisfy the condition $0<\lambda_{1} \leq \lambda_{2} \leq \ldots \rightarrow \infty$.

Thus, differential games for the infinite system of differential Equation (3) are closely related to those for partial differential Equation (1). Therefore, we study differential games for the infinite system of differential Equation (3) separately assuming that $\lambda_{1}, \lambda_{2}, \ldots$ are any numbers. Note that the differential equations we'll consider in the following section correspond to complex numbers $\lambda_{i}$.

\section{Statement of Problem}

We study a differential game for the following system

$$
\begin{array}{ll}
\dot{x_{i}}=-\alpha_{i} x_{i}-\beta_{i} y_{i}+u_{i 1}-v_{i 1}, & x_{i}(0)=x_{i 0}, \\
\dot{y_{i}}=\beta_{i} x_{i}-\alpha_{i} y_{i}+u_{i 2}-v_{i 2}, & y_{i}(0)=y_{i 0},
\end{array}
$$

in Hilbert space $l_{2}$, where $\alpha_{i}, \beta_{i}$ are real numbers, $\alpha_{i} \geq 0,\left(x_{10}, x_{20}, \ldots\right),\left(y_{10}, y_{20}, \ldots\right) \in l_{2}$, pursuer's control parameter $u=\left(u_{1}, u_{2}, \ldots\right)$ and evader's control parameter $v=\left(v_{1}, v_{2}, \ldots\right)$ consist of 2-vectors $u_{i}=\left(u_{i 1}, u_{i 2}\right)$ and $v_{i}=\left(v_{i 1}, v_{i 2}\right), i=1,2, \ldots$, respectively. Throughout the paper we assume that $0 \leq t \leq T$, where $T$ is a sufficiently large number, and $z_{0}=\left(x_{10}, y_{10}, x_{20}, y_{20}, \ldots\right) \neq 0$.

Let $\rho$ and $\sigma$ be given positive numbers.

Definition 1. An admissible control of pursuer is a function $u(t)=\left(u_{1}(t), u_{2}(t), \ldots\right), t \in[0, T]$, whose coordinates $u_{i}(t)$ are measurable and satisfy the condition

$$
\sum_{i=1}^{\infty}\left(u_{i 1}^{2}(t)+u_{i 2}^{2}(t)\right) \leq \rho^{2}, 0 \leq t \leq T .
$$

Definition 2. An admissible control of evader is a function $v(t)=\left(v_{1}(t), v_{2}(t), \ldots\right), t \in[0, T]$, whose coordinates $v_{i}(t)$ are measurable and satisfy the condition

$$
\sum_{i=1}^{\infty}\left(v_{i 1}^{2}(t)+v_{i 2}^{2}(t)\right) \leq \sigma^{2}, 0 \leq t \leq T .
$$

It is assumed that $\rho>\sigma$. Let

$$
z_{i}(t)=\left[\begin{array}{l}
x_{i}(t) \\
y_{i}(t)
\end{array}\right], \quad z_{i 0}=\left[\begin{array}{l}
x_{i 0} \\
y_{i 0}
\end{array}\right], \quad U_{i}=\left[\begin{array}{l}
U_{i 1} \\
U_{i 2}
\end{array}\right], \quad v_{i}=\left[\begin{array}{l}
v_{i 1} \\
v_{i 2}
\end{array}\right],
$$

Definition 3. A strategy of pursuer is a function of the form

$$
U(t, v)=U^{0}(t)+v=\left(U_{1}^{0}(t)+v_{1}, U_{2}^{0}(t)+v_{2}, \ldots\right), \quad U_{i}^{0}(t)=\left(U_{i 1}^{0}(t), U_{i 2}^{0}(t)\right),
$$

where $U^{0}(t)=\left(U_{1}^{0}(t), U_{2}^{0}(t), \ldots\right)$ has measurable coordinates $U_{i}^{0}(t), 0 \leq t \leq T$, that satisfy the condition

$$
\sum_{i=1}^{\infty}\left(\left(U_{i 1}^{0}(t)\right)^{2}+\left(U_{i 2}^{0}(t)\right)^{2}\right) \leq(\rho-\sigma)^{2}, 0 \leq t \leq T .
$$


We denote

$$
A_{i}(t)=e^{-\alpha_{i} t}\left[r r \cos \beta_{i} t-\sin \beta_{i} t \sin \beta_{i} t \cos \beta_{i} t\right], i=1,2, \ldots
$$

We can easily see that the matrix $A_{i}(t)$ has the following properties:

$$
A_{i}(t+h)=A_{i}(t) A_{i}(h), A_{i}^{-1}(t)=A_{i}(-t)=e^{\alpha_{i} t}\left[r r \cos \beta_{i} t \sin \beta_{i} t-\sin \beta_{i} t \cos \beta_{i} t\right] .
$$

Each equation of the system (5) has the unique solution $z(t)=\left(z_{1}(t), z_{2}(t), \ldots\right)$ defined by

$$
z_{i}(t)=A_{i}(t) z_{i 0}+\int_{0}^{t} A_{i}(t-s)\left(u_{i}(s)-v_{i}(s)\right) d s, \quad i=1,2, \ldots
$$

hence,

$$
z_{i}(t)=A_{i}(t)\left[z_{i 0}+\int_{0}^{t} A_{i}(-s)\left(u_{i}(s)-v_{i}(s)\right) d s\right], \quad i=1,2, \ldots
$$

The relations (8) imply that $z(t)=0$ is equivalent to $\eta(t)=0$, where

$$
\eta(t)=\left(\eta_{1}(t), \eta_{2}(t), \ldots\right), \quad \eta_{i}(t)=z_{i 0}+\int_{0}^{t} A_{i}(-s)\left(u_{i}(s)-v_{i}(s)\right) d s, \quad i=1,2, \ldots
$$

Definition 4. We call the number a guaranteed pursuit time if for some strategy of pursuer $U$ and for any admissible control of the evader, $z\left(t^{\prime}\right)=0$ at some $t^{\prime}, 0 \leq t^{\prime} \leq$, where $z(t)=\left(z_{1}(t), z_{2}(t), \ldots\right)$ is the solution of the initial value problem (5).

The pursuer is interested in minimizing the guaranteed pursuit time.

Definition 5. A number $\tau$ is called a guaranteed evasion time if for any number $\tau^{\prime}, 0 \leq \tau^{\prime}<\tau$, we can construct an admissible control $v_{0}(t)$ such that, for the evader such that for any admissible control of the pursuer, we have $z(t) \neq 0$ for all $0 \leq t \leq \tau^{\prime}$ and $i=1,2, \ldots$

The evader is interested in minimizing the guaranteed evasion time.

Problem 1. Find an equation for a guaranteed pursuit time $\theta$ and a guaranteed evasion time $\tau$ in the game (5).

Note that a differential game with integral constraints described by the system (5) was studied in [22]. The present paper dealt with the differential game where the controls of players are subjected to geometric constraints (6) and (7).

\section{Results}

In this section we find a guaranteed pursuit time $\theta$ and construct a strategy for the pursuer that guarantees the time $\theta$.

Theorem 1. The number $\theta$ that satisfy the equation

$$
\sum_{\alpha_{i}>0} \frac{\alpha_{i}^{2}\left|z_{i 0}\right|^{2}}{\sinh ^{2}\left(\alpha_{i} \theta\right)}+\frac{1}{\theta^{2}} \sum_{\alpha_{i}=0}\left|z_{i 0}\right|^{2}=(\rho-\sigma)^{2}
$$

is a guaranteed pursuit time in the game (5).

It should be noted that the series on the left hand side of Equation (6) is a decreasing continuous function of on $(0, \infty)$, approaches $+\infty$ as $\rightarrow 0+$, and approaches 0 as $\rightarrow+\infty$. 
Proof. Let

$$
W_{i}(\theta)=\int_{0}^{\theta} A_{i}(-s) A_{i}^{T}(-s) d s
$$

We have

$$
\begin{aligned}
W_{i}(\theta) & =\int_{0}^{\theta} e^{2 \alpha_{i} s}\left[\begin{array}{cc}
\cos \beta_{i} s & \sin \beta_{i} s \\
-\sin \beta_{i} s & \cos \beta_{i} s
\end{array}\right]\left[\begin{array}{cc}
\cos \beta_{i} s & -\sin \beta_{i} s \\
\sin \beta_{i} s & \cos \beta_{i} s
\end{array}\right] d s \\
& =\int_{0}^{\theta} e^{2 \alpha_{i} s}\left[\begin{array}{ll}
1 & 0 \\
0 & 1
\end{array}\right] d s=\varphi_{i}(\theta) I
\end{aligned}
$$

where

$$
\varphi_{i}(\theta)=\left\{\begin{array}{ll}
\frac{1}{2 \alpha_{i}}\left(e^{2 \alpha_{i} \theta}-1\right), & \alpha_{i} \neq 0 \\
\theta, & \alpha_{i}=0
\end{array}, I=\left[\begin{array}{ll}
1 & 0 \\
0 & 1
\end{array}\right]\right.
$$

We define the strategy for the pursuer as follows

$$
U_{i}(t)= \begin{cases}-A_{i}^{T}(-t) W_{i}^{-1}(\theta) z_{i 0}+v_{i}(t), & 0 \leq t \leq \theta, \\ v_{i}(t), & t>\theta\end{cases}
$$

Note that $U_{i}^{0}(t), i=1,2, \ldots$, in Definition 3 are defined by the equations

$$
U_{i}^{0}(t)=\left\{\begin{array}{ll}
-A_{i}^{T}(-t) W_{i}^{-1}(\theta) z_{i 0}, & 0 \leq t \leq \theta \\
0, & t>\theta
\end{array}, i=1,2, \ldots\right.
$$

To show that the strategy (11) is admissible, we show that

$$
\sum_{i=1}^{\infty}\left|U_{i}^{0}(t)\right|^{2}=\sum_{i=1}^{\infty}\left|-A_{i}^{T}(-t) W_{i}^{-1}(\theta) z_{i 0}\right|^{2} \leq(\rho-\sigma)^{2} .
$$

Indeed, since

$$
-A_{i}^{T}(-t) W_{i}^{-1}(\theta) z_{i 0}=-\frac{e^{\alpha_{i} t}}{\varphi_{i}(\theta)}\left[\begin{array}{cc}
\cos \beta_{i} t & \sin \beta_{i} t \\
-\sin \beta_{i} t & \cos \beta_{i} t
\end{array}\right] z_{i 0},
$$

we have

$$
\begin{aligned}
\left|A_{i}^{T}(-t) W_{i}^{-1}(\theta) z_{i 0}\right| & =\frac{e^{\alpha_{i} t}}{\varphi_{i}(\theta)}\left|\left[\begin{array}{c}
x_{i 0} \cos \beta_{i} t+y_{i 0} \sin \beta_{i} t \\
-x_{i 0} \sin \beta_{i} t+y_{i 0} \cos \beta_{i} t
\end{array}\right]\right| \\
& =\frac{e^{\alpha_{i} t}}{\varphi_{i}(\theta)} \cdot \sqrt{x_{i 0}^{2}+y_{i 0}^{2}} \\
& \leq \frac{e^{\alpha_{i}}}{\varphi_{i}(\theta)} \cdot\left|z_{i 0}\right|= \begin{cases}\frac{\alpha_{i}\left|z_{i 0}\right|}{\sinh \left(\alpha_{i} \theta\right)} & \alpha_{i}>0 \\
\underline{1} & \alpha_{i}=0\end{cases}
\end{aligned}
$$

This implies that

$$
\begin{aligned}
\sum_{i=1}^{\infty}\left|U_{i}^{0}(t)\right|^{2} & =\sum_{i=1}^{\infty}\left|-A_{i}^{T}(-s) W_{i}^{-1}(\theta) z_{i 0}\right|^{2} \\
& \leq \sum_{\alpha_{i}>0} \frac{\alpha_{i}^{2}\left|z_{i 0}\right|^{2}}{\sinh ^{2}\left(\alpha_{i} \theta\right)}+\frac{1}{\theta^{2}} \sum_{\alpha_{i}=0}\left|z_{i 0}\right|^{2} .
\end{aligned}
$$


We are now in position to prove the admissibility of strategy (11). By the Minkowskii inequality and the definition of $\theta$ we have, for $0 \leq t \leq \theta$,

$$
\begin{aligned}
\|U(t)\| & =\left\|U_{0}(t)+v(t)\right\| \leq\left\|U_{0}(t)\right\|+\|v(t)\| \\
& =\left(\sum_{i=1}^{\infty}\left|U_{i}^{0}(t)\right|^{2}\right)^{1 / 2}+\left(\sum_{i=1}^{\infty}\left|v_{i}(t)\right|^{2}\right)^{1 / 2} \\
& \leq\left(\sum_{\alpha_{i}>0} \frac{\alpha_{i}^{2}\left|z_{i 0}\right|^{2}}{\sinh ^{2}\left(\alpha_{i} \theta\right)}+\frac{1}{\theta^{2}} \sum_{\alpha_{i}=0}\left|z_{i 0}\right|^{2}\right)^{1 / 2}+\sigma=\rho-\sigma+\sigma=\rho .
\end{aligned}
$$

The proof of admissibility of strategy $U(t)$ is complete.

To show that $\theta$ is a guaranteed pursuit time, we show that $z(\theta)=0$. To this end we show that

$$
\eta_{i}(\theta)=0, \quad i=1,2, \ldots
$$

Indeed, by (11)

$$
\begin{aligned}
\eta_{i}(\theta) & =z_{i 0}+\int_{0}^{\theta} A_{i}(-s)\left(U_{i}(s)-v_{i}(s)\right) d s \\
& =z_{i 0}+\int_{0}^{\theta} A_{i}(-s)\left(-A_{i}^{T}(-s) W_{i}^{-1}(\theta) z_{i 0}\right) d s \\
& =z_{i 0}-z_{i 0}=0,
\end{aligned}
$$

and so $z_{i}(\theta)=0$, hence, $z(\theta)=0$. Thus, pursuit is completed at the time $\theta$. This completes the proof of the theorem.

\section{Guaranteed Evasion Time}

In this section, we study the evasion differential game and we find a guaranteed evasion time $\tau$. We prove the following statement.

Theorem 2. For any initial state $z_{0}=\left(z_{10}, z_{20}, \cdots\right)$, the number

$$
\tau=\sup _{i} \tau_{i}, \quad \tau_{i}= \begin{cases}\frac{1}{\alpha_{i}} \ln \left(\frac{\alpha_{i}\left|z_{i 0}\right|}{\rho-\sigma}+1\right), & \alpha_{i}>0 \\ \frac{\left|z_{i 0}\right|}{\rho-\sigma}, & \alpha_{i}=0\end{cases}
$$

is a guaranteed evasion time in game (5).

Proof. Let pursuer apply an arbitrary admissible control $u=u(t)$. Let $\tau^{\prime}$ be an arbitrary time that satisfies the condition $0<\tau^{\prime}<\tau$. To prove the theorem, we construct an admissible control for the evader, such that $z(t) \neq 0,0 \leq t<\tau^{\prime}$. Indeed, by definition of $\tau$ there exists $j \in\{1,2, \cdots\}$ such that $\tau^{\prime}<\tau_{j}$. Show that there is a control of the evader such that $z(t) \neq 0,0 \leq t<\tau_{j}$. Observe $z_{j 0} \neq 0$ since otherwise $\tau_{j}=0$ contradicting positivity of $\tau_{j}$. Let $e_{j}=\frac{z_{j 0}}{\left|z_{j 0}\right|}$ and

$$
v_{j}(t)=-\sigma\left[\begin{array}{rr}
\cos \beta_{j} t & \sin \beta_{j} t \\
-\sin \beta_{j} t & \cos \beta_{j} t
\end{array}\right] e_{j}, \quad v_{i}(t)=0, i=1,2, \cdots, i \neq j, \quad t \in\left[0, \tau_{j}\right) .
$$


Then, for any admissible control of the pursuer $u(\cdot)$ and time $t \in\left[0, \tau_{j}\right)$, we have

$$
\begin{aligned}
\eta_{j}(t) & =z_{j 0}+\int_{0}^{t} A_{i}(-s) u_{j}(s) d s-\int_{0}^{t} A_{i}(-s) v_{j}(s) d s \\
& =z_{j 0}+\int_{0}^{t} A_{i}(-s) u_{j}(s) d s+\sigma e_{j} \int_{0}^{t} e^{\alpha_{j} s} d s .
\end{aligned}
$$

From this using the inequality $\left|\left(A_{i}(-s) u_{j}(s), e_{i}\right)\right| \leq \rho e^{\alpha_{j} s}$ and the definition of $\tau_{j}$ we obtain

$$
\begin{aligned}
\eta_{j}(t) e_{j} & =\left|z_{j 0}\right|+\int_{0}^{t}\left(A_{i}(-s) u_{j}(s), e_{i}\right) d s+\sigma \int_{0}^{t} e^{\alpha_{j} s} d s \\
& \geq\left|z_{j 0}\right|-\rho \int_{0}^{t} e^{\alpha_{j} s} d s+\sigma \int_{0}^{t} e^{\alpha_{j} s} d s \\
& >\left|z_{j 0}\right|-(\rho-\sigma) \int_{0}^{\tau_{j}} e^{\alpha_{j} s} d s=0 .
\end{aligned}
$$

Consequently, $\eta_{j}(t) \neq 0$ and hence $z_{j}(t) \neq 0$ by (9), therefore, $z(t) \neq 0$ for $t \in\left[0, \tau_{j}\right)$. In particular, $z(t) \neq 0$ on the interval $\left[0, \tau^{\prime}\right]$. This completes the proof of Theorem 2.

\section{Discussion}

We have studied a pursuit and evasion differential games for an infinite system of differential equations. We have obtained a formula for the guaranteed pursuit time. Additionally, we have constructed an explicit strategy for the pursuer that ensures the completion of the game by the guaranteed pursuit time.

We estimate the guaranteed pursuit time $\theta$. Using the Taylor series, for $\alpha_{i} \neq 0$, we have

$$
\begin{aligned}
\frac{\alpha_{i}}{\sinh \alpha_{i} \theta} & =\frac{2 \alpha_{i}}{e^{\alpha_{i} \theta}-e^{-\alpha_{i} \theta}} \\
& =\frac{2 \alpha_{i}}{1+\alpha_{i} \theta+\frac{\alpha_{i}^{2} \theta^{2}}{2 !}+\frac{\alpha_{i}^{3} \theta^{3}}{3 !}+\cdots-\left(1-\alpha_{i} \theta+\frac{\alpha_{i}^{2} \theta^{2}}{2 !}-\frac{\alpha_{i}^{3} \theta^{3}}{3 !}+\ldots\right)} \\
& =\frac{2 \alpha_{i}}{2 \alpha_{i} \theta\left(1+\frac{\alpha_{i}^{2} \theta^{2}}{3 !}+\frac{\alpha_{i}^{4} \theta^{4}}{5 !}+\ldots\right)} \leq \frac{1}{\theta} .
\end{aligned}
$$

Therefore, we obtain from (10) that

$$
\begin{aligned}
(\rho-\sigma)^{2} & =\sum_{\alpha_{i}>0} \frac{\alpha_{i}^{2}\left|z_{i 0}\right|^{2}}{\sinh ^{2}\left(\alpha_{i} \theta\right)}+\sum_{\alpha_{i}=0} \frac{\left|z_{i 0}\right|^{2}}{\theta^{2}} \\
& \leq \sum_{\alpha_{i}>0} \frac{\left|z_{i 0}\right|^{2}}{\theta^{2}}+\sum_{\alpha_{i}=0} \frac{\left|z_{i 0}\right|^{2}}{\theta^{2}}=\frac{\left|z_{0}\right|^{2}}{\theta^{2}}
\end{aligned}
$$

where

$$
\left|z_{0}\right|=\left(\sum_{i=1}^{\infty}\left|z_{i 0}\right|^{2}\right)^{1 / 2}
$$

Hence,

$$
\theta \leq \frac{\left|z_{0}\right|}{\rho-\sigma}
$$

Thus, the guaranteed pursuit time is less than or equal to $\left|z_{0}\right| /(\rho-\sigma)$. Hence, the strategy of pursuer (11) guarantees the completion of the game by the time $\left|z_{0}\right| /(\rho-\sigma)$.

Additionally, we have obtained a formula for the guaranteed evasion time. 
Author Contributions: Investigation, M.T., G.I., S.K. and R.M.H.; Methodology, M.T., G.I., S.K. and R.M.H.; Project administration, S.K. and R.M.H.; Supervision, G.I.; Validation, M.T., G.I., S.K. and R.M.H.; Writing original draft, M.T., G.I., S.K. and R.M.H.; Writing review and editing, M.T., G.I., S.K. and R.M.H. All authors have read and agreed to the published version of the manuscript.

Funding: The present research was partially supported by the National Fundamental Research Grant Scheme FRGS of Malaysia, FRGS/1/2017/STG06/UPM/02/9.

Institutional Review Board Statement: Not applicable.

Informed Consent Statement: Not applicable.

Conflicts of Interest: The authors declare no conflict of interest.

\section{References}

1. $\quad$ Isaacs, R. Differential Games; John Wiley \& Sons: New York, NY, USA, 1965.

2. Berkovitz, L.D. A Survey of Differential Games, Mathematical Theory of Control; Academic Press: New York, NY, USA, 1967; pp. 373-385.

3. Elliot, R.J.; Kalton, N.J. The Existence of Value for Differential Games; American Mathematical Soc.: Providence, RI, USA, 1972.

4. Fleming, W.H. A note on differential games of prescribed duration. Contrib. Theory Games 1957, 3, 407-416.

5. Fleming, W.H. The convergence problem for differential games. J. Math. Anal. Appl. 1961, 3, 102-116. [CrossRef]

6. $\quad$ Friedman, A. Differential Games; Wiley Interscience: New York, NY, USA, 1971.

7. Hajek, O. Pursuit Games; Academic Press: New York, NY, USA, 1975.

8. Ho, Y.; Bryson, A.; Baron, S. Differential games and optimal pursuit-evasion strategies. IEEE Trans. Autom. Control 1965, 10, 385-389. [CrossRef]

9. Pontryagin, L.S. Selected Works; Nauka: Moscow, Russia, 1988.

10. Krasovskii, N.N.; Subbotin, A.I. Game-Theoretical Control Problems; Springer: New York, NY, USA, 1988.

11. Petrosyan, L.A. Differential Games of Pursuit; World Scientific: Singapore, 1993.

12. Pshenichnii, B.N. Simple pursuit by several objects. Cybern. Syst. Anal. 1976, 12, 484-485. [CrossRef]

13. Subbotin, A.I.; Chentsov, A.G. Optimization of Guaranteed Result in Control Problems; Nauka: Moscow, Russia, 1981.

14. Chikrii, A.A. Conflict-Controlled Processes; Kluwer: Dordrecht, The Netherlands, 1997.

15. Ibragimov, G.I.; Allahabi, F.; Kuchkarov, A.S. A pursuit problem in an infinite system of second-order differential equations. UKR. Math. J. 2014, 65, 1203-1216. [CrossRef]

16. Ibragimov, G.I.; Risman, M.H. Pursuit and Evasion Differential game in Hilbert space. Int. Game Theory Rev. 2010, 12, $239-251$. [CrossRef]

17. Ibragimov, G.; Norshakila, A.R.; Kuchkarov, A.; Fudziah, I. Multi Pursuer Differential Game of Optimal Approach with Integral Constraints on Controls of Players. Taiwan J. Math. 2015, 19, 963-976. [CrossRef]

18. Idham, A.A.; Gafurjan, I.; Askar, R. Evasion Differential Game of Infinitely Many Evaders from Infinitely Many Pursuers in Hilbert Space. Dyn. Games Appl. 2017, 7, 347-359. [CrossRef]

19. Salimi, M.; Ibragimov, G.I.; Siegmund, S.; Sharifi, S. On a fixed duration pursuit differential game with geometric and integral constraints. Dyn. Games Appl. 2016, 6, 409-425. [CrossRef]

20. Ibragimov, G.I. The optimal pursuit problem reduced to an infinite system of differential equation. J. Appl. Math. Mech. 2013, 77, 470-476. [CrossRef]

21. Ibragimov, G.I. Optimal pursuit with countably many pursuers and one evader. Differ. Equ. 2005, 41, 627-635. [CrossRef]

22. Ibragimov, G.I. Optimal pursuit time for a differential game in the Hilbert space $l_{2}$. Sci. Asia 2013, 39, 25-30. [CrossRef]

23. Satimov, N.Y.; Tukhtasinov, M. On Some Game Problems for First-Order Controlled Evolution Equations. Differ. Equ. 2005, 41, 1169-1177. [CrossRef]

24. Satimov, N.Y.; Tukhtasinov, M. Game problems on a fixed interval in controlled first-order evolution equations. Math. Notes 2006, 80, 578-589. [CrossRef]

25. Satimov, N.Y.; Tukhtasinov, M. On Game Problems for Second-Order Evolution Equations. Russ. Math. 2007, 51, 49-57. [CrossRef]

26. Salimi, M.; Ferrara, M. Differential game of optimal pursuit of one evader by many pursuers. Int. J. Game Theory 2019, 48, 481-490. [CrossRef]

27. Azamov, A.A.; Ruziboev, M.B. The time-optimal problem for evolutionary partial differential equations. J. Appl. Math. Mech. 2013, 77, 220-224. [CrossRef]

28. Fattorini, H.O. Time-Optimal control of solutions of operational differential equations. SIAM J. Control 1964, 2, 54-59.

29. Fursikov, A.V. Optimal Control of Distributed Systems, Theory and Applications, Translations of Math. Monographs; American Mathematical Soc.: Providence, RI, USA, 2000.

30. Albeverio, S.; Alimov, S.A. On a time-optimal control problem associated with the heat exchange process. Appl. Math. Optim. 2008, 57, 58-68. [CrossRef]

31. Chaves-Silva, F.; Zhang, X.; Zuazua, E. Controllability of evolution equations with memory. SIAM J. Control Optim. 2017, 55, 2437-2459. [CrossRef] 
32. Philippe, M.; Lionel, R.; Pierre, R. Null controllability of the structurally damped wave equation with moving control. SIAM J. Control Optim. 2013, 51, 660-684. [CrossRef]

33. Lions, J.L. Contrôle Optimal de Systémes Gouvernées par des Equations aux Dérivées Partielles; Dunod: Paris, France, 1968.

34. Osipov, Y.S. The theory of differential games in systems with distributed parameters. Dokl Akad. Nauk SSSR 1975, $223,1314-1317$.

35. Avdonin, S.A.; Ivanov, S.A. Families of Exponentials: The Method of Moments in Controllability Problems for Distributed Parameter Systems; Cambridge University Press: Cambridge, UK, 1995.

36. Butkovskiy, A.G. Theory of Optimal Control of Distributed Parameter Systems; Elsevier: New York, NY, USA, 1969.

37. Chernous'ko, F.L. Bounded controls in distributed-parameter systems. J. Appl. Math. Mech. 1992, 56, 707-723. [CrossRef]

38. Ibragimov, G.I. A Problem of Optimal Pursuit in Systems with Distributed Parameters. J. Appl. Math. Mech. 2002, 66, 719-724. [CrossRef]

39. Tukhtasinov, M. Some problems in the theory of differential pursuit games in systems with distributed parameters. J. Appl. Math. Mech. 1995, 59, 979-984. [CrossRef]

40. Tukhtasinov, M.; Mamatov, M.S. On Pursuit Problems in Controlled Distributed Parameters Systems. Math. Notes 2008, 84, 256-262. [CrossRef]

41. Ladyzhenskaya, O.A. Kraevye Zadachi Matematicheskoy Fiziki; Boundary value Problems of Mathematical Physics: Moscow, Russia, 1973. 